\title{
VI. On spherical radiation and vibrations in conical pipes
}

\section{E.H. Barton D.Sc. F.R.S.E.}

To cite this article: E.H. Barton D.Sc. F.R.S.E. (1908) VI. On spherical radiation and vibrations in conical pipes, Philosophical Magazine Series 6, 15:85, 69-81, DOI: 10.1080/14786440809463748

To link to this article: http://dx.doi.org/10.1080/14786440809463748

曲 Published online: 16 Apr 2009.

Submit your article to this journal $\lceil\pi$

Џll Article views: 3

Q View related articles $₫$

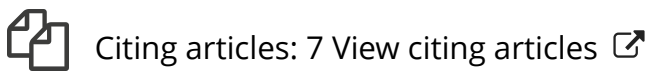


VI. On Spherical Radiation and Vibrations in Conical Pipes. By E. H. Barton, D.Se., F.R.S.E., Professor of Experimental Physics, University College, Nottingham*.

T $\mathrm{T}$ is well known that the vibrations in parallel pipes may be treated by plane waves and elementary methods. When, however, a change is made from parallel to conical pipes the waves cease to be plane, and the method hitherto available is powerless to deal with the phenomena. Yet the importance of the subject is at least equal to that of parallel pipes, since the brass instruments in musical use are conical or quasi-conical, and also the oboe, bassoons, and English horn. Thus, apart from a knowledge of spherical radiation and its application to such pipes, the student is left without a clue to the phenomena occurring. He is accordingly somewhat at a loss to understand why a conical pipe, closed at the vertex and open at the base, should have the same pitch and the same complete series of harmonic tones as a parallel pipe open at both ends. Whereas, a parallel pipe if closed at one end falls in pitch about an octave and loses all the evenly-numbered partials.

The mathematical aspect of the matter is of course treated with great generality and elegance in the classical treatises (see, for example, Rayleigh's 'Theory of Sound,' vol. ii. chaps. xi., xii., \& xiv.). But the use in such treatises of the velocity potential as the dependent variable, slight as this obstacle is, may prove sufficient to prevent some readers from assimilating the articles in question. If', however, following Riemann $t$, we take for the dependent variable the so-called condensation, which is a more familiar conception, the analysis is somewhat simplified and the whole problem is solved by methods within the range of every one familiar with the elements of the calculus. It seems desirable, therefore, that the physical student should be provided with a treatment intermediate between the recondite mathematical treatises on the one hand, and the mere statement of the musical facts respecting conical pipes on the other hand. This plan, already found useful in dealing with such students, is here given in the hope it may thus prove of service to others. Another matter which seems very puzzling, in the simple statement without proof, is the fact that in a conical pipe the anti-nodes remain in equidistant positions as for a parallel pipe, but that the nodes are all shifted, some considerably and others slightly.

* Communicated by the Author.

+ Partielle Differentialgleichungen und deren Anvendung auf physikalische Fragen. 1882. Sixth part. 
This point is here illustrated graphically, the reason for the shifts which occur and their relative amounts being made clear in a single diagram.

Fundamental Assumptions.-In attacking the problem of the spherical radiation of sound in air we simplify the analysis by making at the outset the following assumptions:-

(1) That the action of gravity is negligible.

(2) That the effect of viscosity is negligible.

(3) That the motion is vibratory and devoid of rotation.

(4) That the vibrations are small, so that writing the density

$$
\rho=\rho_{0}(1+s)
$$

the "condensation" $s$ is to be regarded as a small quantity often negligible in comparison with unity.

(5) That the velocities and accelerations of the air are small quantities whose squares and products are negligible.

It is easily seen that the above assumptions simply narrow down the discussion to the case in question, and do not involve the loss of any generality we wish to retain.

In estimating the acceleration of the air two methods are open to us: (a) We may follow in thought an individual particle and note how much its velocity is increased per second; or: (b) We may fix attention on a spot in space and note how the speed changes of that particle (whichever it is) which is found there at the time in question. In other words, we may note the increase of speed of an individual in the procession, or the increase of speed of the procession as it passes a fixed point on the route. The relation between the two accelerations is given in hydrodynamical treatises. In our use of acceleration the first form should in strictness be taken, but, with the limitation (5), the distinction drops, as the difference is only of the second order of small quantities. Thus the second form, which is simpler, may always be used.

We have now to derive the differential equation for aerial vibrations in space of three dimensions, solve it and simplify to the case of spherical radiation, then apply the solution to the various cases of conical pipes.

The differential equation is based upon (i.) the so-called equation of continuity, and (ii.) the equations of motion.

These we now take in the above order.

Equation of Continuity. - Consider an infinitesimal parallelepiped of edges $d x, d y$, and $d z$, and let the velocities of the air parallel to the axes of $x, y$, and $z$ be denoted by $u, v$, and $w$ respectively. 
Then the mass of air entering the parallelepiped at the face $d y d z$ in the time $d t$ is $\rho u(d y d z) d t$, and that leaving by the opposite face in the same time is

$$
\left\{\rho u+\frac{d(\rho u)}{d x} d x\right\} d y d z d t .
$$

Hence, the mass lost by this pair of faces is

$$
\frac{d(\rho u)}{d x} d x d y d z d t
$$

Therefore, considering the other four faces in like manner, we have as the total mass lost by the parallelepiped in time at the quantity

$$
\left\{\frac{d(\rho u)}{d x}+\frac{d(\rho v)}{d y}+\frac{d(\rho w)}{d z}\right\} d x d y d z d t .
$$

But this quantity can also be expressed in terms of the decrease of density, viz. :

$$
-\frac{d \rho}{d t} d x d y d z d t
$$

Whence, equating the two forms, we have

$$
\frac{d \rho}{d t}+\frac{d(\rho u)}{d x}+\frac{d(\rho v)}{d y}+\frac{d(\rho w)}{d z}=0 . . .
$$

Now, since $\rho=\rho_{0}(1+s)$, the first term of $(1)$ becomes $\rho_{0} d s / d t$. Again,

$$
\frac{d(\rho u)}{d x}=\rho_{0}(1+s) \frac{d u}{d x}+u \frac{d \rho}{d x}=\rho_{0} \frac{d u}{d x} \text { nearly, }
$$

the product of the two small quantities $u$ and $d \rho / d x$ being negligible. Thus for our case (1) becomes

$$
\frac{d s}{d t}+\frac{d u}{d x}+\frac{d v}{d y}+\frac{d w}{d r}=0, \quad . \quad . \quad .
$$

and this is the form of the equation of continuity for small oscillations of a light elastic fluid.

Equations of Motion.-We have now to express, for the fluid in our parallelepiped, the condition that the product, mass into acceleration, equals the moving force to which it is subjected. The mass into acceleration is $(\rho d x d y d z) d u / d t$. The moving force is the excess of that due to pressure, $p$, 
behind over that due to pressure, $p+d p$, in front, or

$$
p d y d z-\left(p+\frac{d p}{d x} d x\right) d y d z=-\frac{d p}{d x} d x d y d z .
$$

Hence, equating, we find $-d p / d x=\rho d u / d t$, or

$$
\frac{1}{\rho} \frac{d p}{d u}=-\frac{d u}{d t} \cdot \text {. . . . . . }
$$

But $p$ is some function of $\rho$, and, whatever the form of the curve coordinating $p$ and $\rho$, the small portion with which we are concerned may be considered straight. Hence, for our small vibrations, we can write

$$
d p / d \rho=a^{2}, \text { some constant. }
$$

We can easily see, by the method of dimensions, that $a$ in (4) is of the nature of a velocity. For the left side of (4) is dimensionally

$$
\frac{\mathrm{MLT} \mathrm{T}^{-2} \mathrm{~L}^{-2}}{\mathrm{ML}^{-3}}=\mathrm{L}^{2} \mathrm{~T}^{-2}=(\text { velocity })^{2},
$$

in which $\mathrm{M}, \mathrm{L}$, and $\mathrm{T}$ represent mass, length, and time respectively. From (4) we get

$$
\frac{d p}{\rho}=\frac{a^{2} d \rho}{\rho}=\frac{a^{2} \rho_{0} d s}{\rho_{0}(1+s)}=a^{2} d s \text { nearly. . . }
$$

It should be noted here that we are not entitled to integrate (4) and draw from such result any conclusions about the general relation between $p$ and $\rho$. On the contrary, the relation between them must be determined independently, and the general value of $d p / d \rho=a^{2}$ derived from it.

Substituting (5) in (3), we obtain

$$
a^{2} d s / d x=-d u / d t \text {. }
$$

Whence, for the other axes, we have by symmetry of notation

$$
\text { and } \quad \begin{array}{lllll}
a^{2} d s / d y=-d v / d t, & . & . & . & . \\
a^{2} d s / d z=-d w / d t . & . & . & . & .
\end{array}
$$

These three constitute the required equations of motion.

The Differential Equation. - We have now to derive from equations (2), (6), (7), and (8) the partial differential equation. We see that in addition to $s, x, y, z$, and $t$, the only variables we wish to retain, the above equations involve also $u, v$, and $w$. These last three must accordingly be 
eliminated. On differentiating equation (2) with respect to time, we obtain

$$
-\frac{d^{2} s}{d t^{2}}=\frac{d^{2} u}{d x d t}+\frac{d^{2} v}{d y} v+\frac{d^{2} w}{d t}+\frac{.}{d z d t} .
$$

Again, if we differentiate equation (6) to $x,(7)$ to $y$, and (8) to $z$, and then add the results, we have

$$
-a^{2}\left(\frac{d^{2} s}{d x^{2}}+\frac{d^{2} s}{d y^{2}}+\frac{d^{2} s}{d z^{2}}\right)=\frac{d^{2} u}{d t d x}+\frac{d^{2} v}{d t d y}+\frac{d^{2} w}{d t \overline{d z}} .
$$

But since $d^{2} u / d x d t=d^{2} u / d t d x$, because the order of partial differentiation is indifferent, the right sides of (9) and (10) are identical. Thus, equating their left sides, we have

$$
\left.\begin{array}{rl}
\frac{d^{2} s}{d t^{2}} & =a^{2}\left(\frac{\overrightarrow{d^{2} s}}{d x^{2}}+\frac{d^{2} s}{d y^{2}}+\frac{d^{2} s}{d z^{2}}\right) \\
\text { or } \quad & \frac{d^{2} s}{d t^{2}}=a^{2} \nabla^{2} s,
\end{array}\right\}, . .
$$

where for brevity's sake $\nabla^{2}$ denotes $\frac{d^{2}}{d x^{2}}+\frac{d^{2}}{d y^{2}}+\frac{d^{2}}{d z^{2}}$.

This is the general form of the differential equation applicable to small vibratory disturbances of ' a light compressible medium in space of three dimensions.

Plane Waves.-As a check upon this result, let us reduce it to the case of plane waves in the plane of $y z$, and proceeding therefore in the direction $x$. Thus $s$ is a function of $t$ and $x$ only and is independent of $y$ and $z$. Hence $\nabla^{2} s=d^{2} s / d x^{2}$ and (11) becomes

$$
\frac{d^{2} s}{d t^{2}}=a^{2} \frac{d^{2} s}{d x^{2}}, \ldots . \quad \text {. . . . }
$$

which is the well-known form of equation for plane waves. To examine if $a$ has the right value here let the specific volume be $\mathrm{U}$, then $\rho=1 / \mathrm{U}$ and $d \rho=-d \mathrm{U} / \mathrm{U}^{2}$.

$$
\therefore a^{2}=\frac{d p}{d \rho}=\left(\frac{-d p}{d \mathrm{U} / \mathrm{U}}\right) \mathrm{U}=\mathrm{EU}=\mathrm{E} / \rho=\gamma p / \rho, .
$$

in which $\mathrm{E}$ denotes the volume elasticity and $\gamma$ the ratio of the two specific heats. Thus, the ordinary value is seen to hold.

Solution for Spherical Waves.-Let us now transform to polar coordinates defined by

$x=r \sin \theta \cos \phi, \quad y=r \sin \theta \sin \phi, \quad$ and $\quad z=r \cos \theta$. 
Then it is shown in text-books on the calculus (e.g. Williamson's 'Differential Calculus') that

$$
\nabla^{2} s=\frac{d^{2} s}{d r^{2}}+\frac{2}{r} \frac{d s}{d r}+\frac{1}{r^{2} \sin \theta} d \dot{d} \theta\left(\sin \theta \frac{d s}{d \bar{\theta}}\right)+\frac{1}{r^{2} \sin ^{2} \theta} \frac{d^{2} s}{d \phi^{2}} .
$$

Now suppose we have symmetry about the centre of the system, so that $s$ is a function of $r$ only. Then $d s / d \theta=0$ and $d s / d \phi=0$. Hence, for this case, (15) reduces to

$$
\nabla^{2} s=\frac{d^{2} s}{d r^{2}}+\frac{2}{r} \frac{d s}{d r}=\frac{1}{r} \frac{d^{2}(r s)}{d r^{2}} . \quad \text {. . . . }
$$

Equation (11) accordingly becomes

$$
a^{2} \frac{d^{2}(r s)}{d r^{2}}=\frac{d^{2}(r s)}{d t^{2}}
$$

The general solution of this may be written

$$
r s=f_{1}(r-a t)+f_{2}(r+a t), . \quad . \quad .
$$

where $f_{1}$ and $f_{2}$ denote arbitrary functions. This solution obviously consists of both djverging and converging waves of spherical form, of any periodic or non-periodic type and travelling with radial speed $a$. For diverging waves of simple harmonic type (18) becomes

$$
s=\frac{c}{r} \cos k(r-a t) . \quad . \quad . \quad . \quad .
$$

To denote the speed $u$ of the air along the radius $r$, we derive from (6) and (19)

$\frac{-d u}{d t}=a^{2} \frac{d s}{d r}=\frac{a^{2} c}{r}\left\{-k \sin k(r-a t)-\frac{1}{r} \cos k(r-a t)\right\}$.

Thus

$$
u=-a^{2} \int \frac{d s}{d r} d t=\frac{c a}{r}\left\{\cos k(r-a t)-\frac{1}{k r} \sin k(r-a t)\right\} .
$$

Again, if displacements along $r$ be denoted by $\xi$, we have by another integration

$$
\begin{gathered}
\xi=\int u d t, \text { or } \\
\xi=-\frac{c}{k r}\left\{\sin k(r-a t)+\frac{1}{k r} \cos k(r-a t)\right\} .
\end{gathered}
$$

We may thus see, from equations (19), (21), and (22), that it is only the condensation $s$ whose change of phase is restricted to the ordinary one inseparably associated with the 
advance of a progressive wave. Its amplitude, however, suffers diminution by varying inversely as $r$. But, owing to this diminution or attenuation with advance, we have in the other equations the factors $1 / r$ and also $1 / r^{2}$, one applying to a sine and the other to a cosine function. Hence the speed $u$ and the displacement $\xi$ each exhibit, during advance, an additional slight change of phase (as the sine and cosine terms are differently diminished), beyond that always present in a progressive wave. But, where $r$ is great enough to make $1 / k r$ negligibly small, (21) and (22) become approximately

$$
\text { and } \quad \begin{aligned}
u & =\frac{c a}{r} \cos k(r-a t), \ldots . \quad . \quad . \\
\xi & =\frac{-c}{k r} \sin k(r-a t) . \quad . \quad . \quad .
\end{aligned}
$$

Reflexion at Pole.-Let us now regard the two spherical waves of (18) as a converging one and a diverging one to which the other gives rise by reflexion at the pole or centre of the system. And let it be required to determine the relation between $f_{1}$ and $f_{2}$ so resulting. The total current across the surface of a sphere of radius $r$ is $4 \pi r^{2} u$ and, for $r=0$, must vanish, since all is symmetrical about the pole or origin of coordinates. That is, $u$ cannot be infinite and so make $r^{2} u$ finite for $r=0$. But, if $4 \pi r^{2} u$ vanishes at $r=0$ for all values of $t$, so also will $4 \pi r^{2} d u / d t$ vanish. And this condition is easier to fulfil analytically. Thus from (6) and (18) we have

$$
\begin{gathered}
4 \pi r^{2} d u / d t=-4 \pi a^{2} r^{2} d s / d r^{2} \\
=4 \pi a^{2}\left\{f_{1}(r-a t)+f_{2}(r+a t)\right\}-4 \pi a^{2} r\left\{f_{1}^{\prime}(r-a t)+f_{2}^{\prime}(r+a t)\right\},
\end{gathered}
$$

where $f^{\prime}$ denotes the first derived function of $f$.

Hence, putting $d u / d t=0$ for $r=0$, we find

$$
0=f_{1}(-a t)+f_{2}(a t) \text {. . . . . . }
$$

as the relation between $f_{1}$ and $f_{2}$. But we see from (18) that the right side of (25) is the value of $(r s)$ for $r=0$. Hence, we may write as the condition at the pole

$$
r s=0 \text { for } r=0, . \text {. . . . . }
$$

or, $r s$ must vanish with $r$.

Thus, at the pole, a condensation is reflected as a rarefaction and vice versa, somewhat as in the case of reflexion at the open end of a parallel pipe.

Conical Pipes.-To apply equation (17) to conical pipes 
we must choose a form of solution corresponding to stationary waves. Thus, let $r s$ be everywhere proportional to cos kat. Then $d^{2}(r s) / d t^{2}=-k^{2} a^{2}(r s)$, and equation (17) transforms into

$$
\frac{d^{2}(r s)}{d r^{2}}+k^{2}(r s)=0 . \quad \text {. . . . . . }
$$

The general solution of this may be written

$$
r s=(\mathrm{A} \cos k r+\mathrm{B} \sin k r) \cos k a t, \ldots .
$$

where $A$ and $B$ are arbitrary constants. These are to be determined for each case by the position and nature of the ends of the pipe. There are accordingly a number of separate cases to consider.

Open Ends.-First let both ends of the conical pipe be open. Then obviously the condition at the end is approximately $s=0$. For, at the ideal open end there can be neither condensation nor rarefaction. Let the coordinates of the ends of the pipe be $r_{1}$ and $r_{2}$, measured from the vertex of the cone if completed. Then, we have from (28) for the terminal conditions,

$\mathrm{A} \cos k r_{1}+\mathrm{B} \sin k r_{1}=0$ and $\mathrm{A} \cos k r_{2}+\mathrm{B} \sin k r_{2}=0$;

whence, by the elimination of $\mathrm{A} / \mathrm{B}$, we obtain

$$
\sin k\left(r_{2}-r_{1}\right)=0 \text { or } k\left(r_{2}-r_{1}\right)=n \pi \text {. }
$$

This may be written

$$
r_{2}-r_{1}=n \lambda_{n} / 2 \quad \text { or } \quad \mathrm{N}_{n}=\frac{n a}{2\left(r_{2}-r_{1}\right)}, \quad .
$$

where $n$ is an integer ; for since $s$ is proportional to $\cos \dot{k} a t$, $\dot{b}=2 \pi \mathrm{N} / a=2 \pi / \lambda, \mathrm{N}$ being the frequency and $\lambda$ the wavelength of the motion. Thus, for a conical pipe with open ends, the pitch of the prime tone and the form of the series of other nitural tones are like those for an open-ended parallel pipe. This might have been anticipated from the similarity of the differential equations and the conditions for the open ends in each case, There is, however, this slight difference that $r_{2}-r_{1}$ is the slant iength of the conical pipe and not its axial length. As to the segments into which the pipe is divided when emitting its higher natural tones, it follows from equation (29) that the antinodes are equidistant. When dealing with the next case, it will be seen that this simplicity does not extend to the nodes.

Closed Ends. - The condition at closed ends is obviously $u=0$; consequently $d u / d t=0$ there also. But by equation (6) $d u / d t=a^{2} d s / d r$, if $u$ denotes the velocity along $r$. We may thus write as our condition for a closed end $d s / d r=0$. 
Applying this to equation (28), we find, after a little transformation,

$$
\mathrm{A}\left(\cos k r_{1}+k r_{1} \sin k r_{1}\right)=\mathrm{B}\left(k r_{1} \cos k r_{1}-\sin k r_{1}\right),
$$

and $\quad \mathrm{A}\left(\cos k r_{2}+k r_{2} \sin k r_{2}\right)=\mathrm{B}\left(k r_{2} \cos k r_{2}-\sin k r_{2}\right)$.

On dividing out by the cosines and writing $\tan \theta_{1}$ for $k r_{1}$ and $\tan \theta_{2}$ for $k r_{2}$, we may eliminate $\mathrm{A} / \mathrm{B}$ between the above equations. Thus

$$
\begin{aligned}
\mathrm{A} / \mathrm{B}=\frac{\tan \theta_{1}-\tan k r_{1}}{1+\tan \theta} \theta_{1} \tan k r_{1} & =\tan \left(\theta_{1}-k r_{1}\right)=\tan \left(\theta_{2}-k r_{2}\right), \\
k r_{2}-\tan ^{-1} k r_{2} & \left.=k r_{1}-\tan ^{-1} k r_{1} . \quad . \quad . \quad . \quad \text { or } \quad 30\right)
\end{aligned}
$$

The transcendental form of this equation shows that the nodes are not equidistant in a conical pipe. We will presently find where they are in the important case of a complete cone with open end.

Closed Cone.-To treat the case of a cone continued to the vertex and with base closed, we have simply to write $r_{1}=0$ in equation (30) and $R$ as the slant length of the cone for $r_{2}$. This gives

$$
\tan k \mathrm{R}=k \mathrm{R} \text {. }
$$

To solve this equation, which we may regard as $\tan x=x$, we may proceed graphically. Thus plot the two graphs $y=x$ and $y=\tan x$. Then their intersections will give the roots required. See fig. 1, p. 80 as an illustration of this. The equation may also be solved by successive approximations by which (in another connexion) Lord Rayleigh finds ("Theory of Sound,' vol. i. p. 334),

$$
\begin{aligned}
& \left.\frac{k \mathrm{R}}{\pi}=x / \pi=0,1 \cdot 4303,2 \cdot 4590,3 \cdot 4709,4 \cdot 4747,5 \cdot 4818,6 \cdot 4844, \& c .\right\} \\
& \left.=\theta_{1}, \quad \theta_{2}, \quad \theta_{3}, \quad \theta_{1}, \quad \theta_{5}, \quad \theta_{6}, \quad \theta_{7}, \text { say, }\right\}
\end{aligned}
$$

Thus these quantities, denoted by the $\theta^{\prime}$ s, each multiplied by $\pi$, give the first seven values of $k R$ in equation (31).

Now since $\theta_{1}=0$, we may write $\left(k_{n} \mathrm{R}\right)=\pi \theta_{n+1}$. But we also have as the general relation $k_{n}=2 \pi \mathrm{N}_{n} / a$. Hence, we may write for the frequency of the $n$th tone natural to the closed cone

$$
\mathrm{N}_{n}=\frac{a}{2 \mathrm{R}} \theta_{n+1} \ldots . \quad . \quad . \quad . \quad .
$$

Thus the frequencies are directly proportional to the speed of sound, inversely proportional to the slant length of the cone and the relation of the various possible tones in the series is defined by equation (32) giving the roots of (31). 
Open Cone.-We now consider the case of a complete cone with base open. At the open base, we have, as before for an open end, the condition $r s=0$. And at the vertex, the origin of coordinates, we have from equation (26) that $r s=0$ there also. So that although one end is open and the other closed, we have the apparent anomaly that the same condition applies to each. Hence if $R$ is the coordinate of the base, $i, e . \mathbf{R}$ is the slant length of the cone, we have from (29)

$$
\mathrm{R}=m \lambda_{m} / 2 \quad \text { or } \quad \mathrm{N}_{m}=m a / 2 \mathrm{R}, \quad . \quad . \quad
$$

where $\mathrm{N}_{m}$ is the frequency of the $m^{\text {th }}$ natural tone and $\lambda_{m}$ its wave-length, $a$ being the speed of sound. This then shows, what has before been remarked upon as strange, that a cone open at the base and closed at the vertex gives practically the same fundamental and the same full harmonic series of other natural tones as are obtainahle from a parallel pipe of the same length and open at both ends. Of course, when the corrections for open ends are taken into account, the statement as to pitch and length suffers a slight modification. For the double open-ended parallel pipe has two ends needing correction and the cone only one; moreover their diameters may differ. But if a cone and an open-ended parallel pipe are prepared of slightly different lengths so that their fundamentals are in unison, then their other partials will be in accord also. This may easily be verified by pipes of zinc tested with a set of tuning-forks forming the harmonic series of relative frequencies $1,2,3,4$, \&e.

We see from the first form of (34) that the wave-length is inversely as the order of the tone produced; hence the antinodes are all equidistant. This, however, does not apply to the nodes. To determine the positions of the nodes we must refer to equations (31) to (34). Now equation (33) gives in terms of $\theta$ the various values of $\mathrm{N}$ for a closed cone of fixed slant length $\mathbf{R}$. Let us, however, substitute the variable $r$ for the constant $\mathrm{R}$ and, dropping the subscript of $\mathrm{N}$, rewrite this equation as follows :-

$$
2 \mathrm{~N} r / a=\theta_{1}, \theta_{2}, \theta_{3}, \theta_{4}, \theta_{5}, \theta_{6} \text {, or } \theta_{7} \text {, \&c. . . }
$$

We may now regard both $N$ and $r$ as variables which must satisfy $(35), r$ being the slant length of a closed cone. Again, equation (34) gives the frequencies of the various tones natural to the open cone. Let us rewrite it, dropping from $\mathrm{N}$ its subscript and writing for $m$ on the right side the series of natural numbers which it represents. We thus obtain

$$
2 \mathrm{NR} / a=1,2,3,4,5,6 \text {, or } 7, \text { de. . . }
$$


Here we consider the slant length $\mathrm{R}$ of our open cone as a constant and $\mathrm{N}$ to vary in accordance with the numbers on the right side of the equation. Now, on dividing equation (35) by (36) we eliminate the frequency $\mathrm{N}$ and obtain the required relations between $r$ and $R$, viz.:-

$$
\left.\begin{array}{r}
\stackrel{r}{\mathrm{R}}=-\frac{\theta_{1}}{1}, \frac{\theta_{1} \text { or } \theta_{2}}{2}, \frac{\theta_{1}, \theta_{2} \text { or } \theta_{3}}{3}, \frac{\theta_{1}, \theta_{2}, \theta_{3} \text { or } \theta_{4}}{4}, \frac{\theta_{1}, \theta_{2}, \theta_{3}, \theta_{4} \text { or } \theta_{5}}{5}, \\
\frac{\theta_{1}, \theta_{2}, \theta_{3}, \theta_{4}, \theta_{5} \text { or } \theta_{6}}{6}, \text { or } \frac{\theta_{1}, \theta_{2}, \theta_{3}, \theta_{4}, \theta_{5}, \theta_{6} \text { or } \theta_{7}}{7}, \& \mathrm{c} .
\end{array}\right\}
$$

It is necessary to cross combine the right sides of (35) and (36) in this way to obtain all the values sought. The denominator of any one of the fractions on the right of (37) shows the order of the tone being enitted by the pipe. The various values of $r / \mathrm{R}$ obtained by taking the various $\theta$ 's in the numerator of that fraction locate the nodes for the tone in question. The series of $\theta$ 's in each numerator is finite, being limited by the obvious fact that $r / \mathbf{R}$ cannot exceed unity. The first few nodal positions are given in Table I. They are also exhibited graphically together with the positions of the

\begin{tabular}{|c|c|c|c|c|c|c|c|}
\hline $\begin{array}{l}\text { Order } m \text { of } \\
\text { Natural Tone; } \\
\text { i. e. denominator } \\
\text { of fraction on } \\
\text { right side of }\end{array}$ & & i. e., $\mathrm{v}$ & lues of & $\begin{array}{l}\text { al Positi } \\
R=\theta / m\end{array}$ & equatio & (37). & \\
\hline $1 \ldots \ldots \ldots \ldots$ & 0 & & & & & & \\
\hline $2 \ldots \ldots \ldots \ldots$ & 0 & 07152 & & & & & \\
\hline $3 \ldots \ldots \ldots \ldots$ & 0 & $0 \cdot 4768$ & $0 \cdot 8197$ & & & & \\
\hline $4 \ldots \ldots \ldots \ldots$ & 0 & $0 \cdot 3576$ & $0 \cdot 6148$ & 0.8677 & & & \\
\hline $5 \ldots \ldots \ldots$ & 0 & 0.2861 & 0.4918 & 0.6942 & 0.8949 & & \\
\hline 6 & 0 & $0 \cdot 2384$ & 0.4098 & 0.5785 & 077458 & 0.9136 & \\
\hline $7 \ldots \ldots \ldots \ldots$ & 0 & 0.2043 & 0.3513 & 0.4958 & $0 \cdot 6392$ & 0.7831 & 0.9263 \\
\hline $\begin{array}{c}\text { Values of } \theta \text { 's in } \\
\text { numerators of } \\
\text { fractions on right } \\
\text { side of equation (37) }\end{array}$ & $\begin{array}{r}\theta_{1} \\
0\end{array}$ & $\begin{array}{c}\theta_{2} \\
1 \cdot 4303\end{array}$ & $\begin{array}{c}\theta_{3} \\
2 \cdot 4590\end{array}$ & $\begin{array}{c}\theta_{4} \\
3 \cdot 4709\end{array}$ & $\begin{array}{c}\theta_{5} \\
4 \cdot 4747\end{array}$ & $\begin{array}{c}\theta_{6} \\
5 \cdot 4818\end{array}$ & $\begin{array}{c}\theta_{7} \\
6.4844\end{array}$ \\
\hline
\end{tabular}

Table I.-Nodal Positions of an Open Cone. 
80 Spherical Radiation and Vibrations in Conical Pipes.

antidodes in fig. 1. In this diagram the graph $y=x$ is shown by a full line and the branches of $y=\tan x$ by broken lines. The abscissæ of their intersections show the values of the roots of the equation, $\tan x=x$.

FIG. 1.-Vibrating Segments of an Open Cone.

The circles show the equidistant antinodes and the crosses show the nodes loeated by the intersections of $x=y$ and $y=\tan x$.

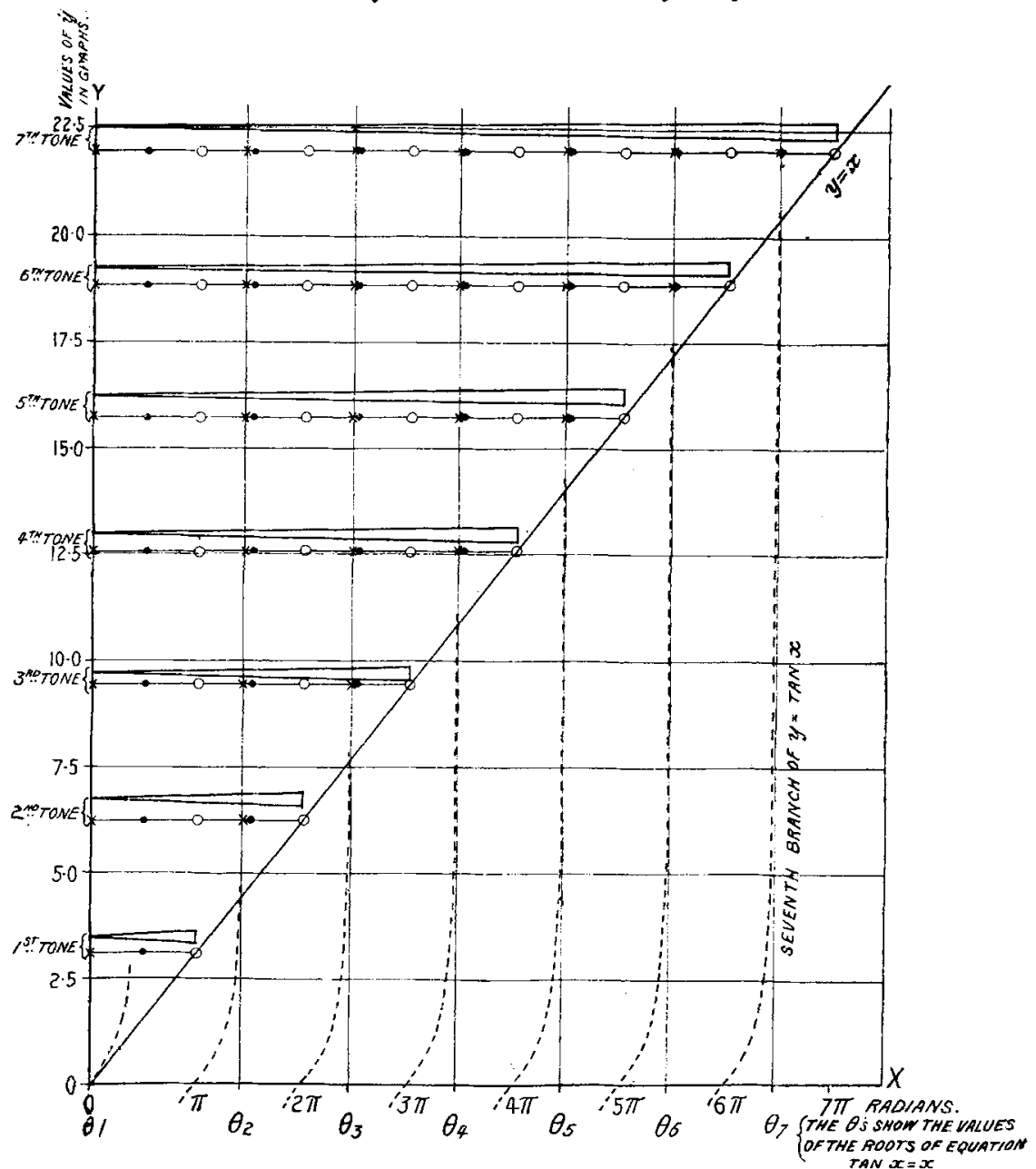

To show the segments of one pipe of fixed length when emitting its various tones, a number of such diagrams would be needed. To avoid this repetition, a series of pipes is shown 
in the upper part of the diagram. These are of various length so that the longest at the top is shown with the segments corresponding to its seventh natural tone. Passing down the series, we reach the last pipe, the shortest of all, with one segment only as when emitting its fondamental. The antinodes are indicated by small circles and the nodes by crosses. The equidistant positions midway between the antinodes are shown by dots.

Experiments by Mr. D. J. Blaikley have shown the existence of these nodes in positions slightly displaced from equidistances, each node being moved towards the vertex from the corresponding equidistant position. Further, it was found by Blaikley that the displacement was the greater for those nodes which are nearer the vertex. Or, in other words, "the nodes in the cone are at increasing distances apart, reckoning from the open end, and at the apex of the cone is a node common to all the notes." These nodal positions were established by Blaikley with an experimental bugle made in sections. On taking this to pieces, thin metal diaphragms were inserted at the positions of the nodes for a certain note, and their presence was found not to prevent the production of the note in question. The diaphragms had each a few small holes to admit the passage of the player's breatb, but prevented all free vibration at the place.

University College, Nottingham, June $7,1907$.

VII. On the Rupture of Materials under Combined Stress :Tension and Hydrostatic Pressure. By W. ELLIs Williams, B.Sc., Research Fellow of the University of Wales*.

TWHE connexion between the rupture of a solid body and 1 the stress causing it is at present but vaguely understood, and a number of different theories have been put forward to enable the tendency to rupture to be calculated from the stresses acting on the body by the methods of elastic theory. The principal of these are $\dagger$ :-

(1) LAME.-Rupture occurs when the greatest tension has reached a certain limit.

(2) Poncelet and St. Venast.-Rupture occurs when the greatest extension has reached a certain limit.

* Communicated by Prof. E. Taylor Jones.

† Love, 'Elasticity,' vol. i. p. 106.

Phil. Mag. S. 6. Vol. 15. No. 85. Jan. 1908. 